\title{
Cancer incidence in people with affective disorder: nationwide cohort study in Taiwan, 1997-2010
}

Yen-Ni Hung, Shu-Yu Yang, Ming-Chyi Huang, For-Wey Lung, Shih-Ku Lin, Kuan-Yu Chen, Chian-Jue Kuo* and Ying-Yeh Chen*

\section{Background}

Cancer is a serious public health problem worldwide, and its relationship with affective disorders is not clear.

\section{Aims}

To investigate alcohol- and tobacco-related cancer risk among patients with affective disorders in a large Taiwanese cohort.

\section{Method}

Records of newly admitted patients with affective disorders from January 1997 through December 2002 were retrieved from the Psychiatric Inpatient Medical Claims database in Taiwan. Cancers were stratified by site and grouped into tobacco- or alcohol-related cancers. Standardised incidence ratios (SIRS) were calculated to compare the risk of cancer between those with affective disorders and the general population.

\section{Results}

Some 10207 patients with bipolar disorder and 9826 with major depression were included. The risk of cancer was higher in patients with major depression $(\mathrm{SIR}=2.01$, 95\% Cl 1.85-2.19) than in those with bipolar disorder (SIR 1.39, 95\% Cl 1.26-1.53). The elevated cancer risk among individuals ever admitted to hospital for affective disorders was more pronounced in tobacco- and/or alcohol-related cancers.

\section{Conclusions}

Elevated cancer risk was found in patients who had received in-patient care for affective disorders. They require holistic approaches to lifestyle behaviours and associated cancer risks.

\section{Declaration of interest}

None.
Affective disorders such as bipolar disorder and major depressive disorders are recurring mental illnesses complicated by high comorbidity and risk of poor health outcomes. ${ }^{1,2}$ Even though cancer has become a serious public health problem worldwide, studies have not consistently demonstrated a positive relationship between affective disorders and subsequent risk of cancer. ${ }^{3}$ There are several explanations for the link between affective disorders and cancer incidence. First, people with affective disorders typically have an unhealthy lifestyle, ${ }^{4}$ which could be related to their vulnerability to cancer, such as tobacco smoking and alcohol use; these substances are identified as avoidable risk factors for cancer. $^{5-8}$ Second, affective disorders cause alterations in immune pathways and prompt inflammatory responses, predisposing individuals to immune-related diseases such as cancer. In addition to people with affective disorders having been demonstrated to have a marked increase in microglial and circulating monocyte activation, ${ }^{9}$ affective symptoms have moreover been associated with markers of increased inflammation (such as high levels of interleukin-1 receptor antagonist (IL-1Ra), high-sensitivity C-reactive protein (hs-CRP) and soluble tumour necrosis factor receptor 1 (sTNF-R1)). ${ }^{10}$ As some of these markers have been noted to persist even in full remission, it is possible that some patients with affective disorders may experience chronic inflammation. ${ }^{10}$ Third, due to genetic heterogeneity, bipolar disorder and major depression could be risk factors of cancer. Indeed, several epidemiological studies support positive associations between bipolar disorders and major depressive disorders with subsequent cancer risk. ${ }^{11-13}$ Some studies, however, showed otherwise..$^{8,14,15}$ One possible explanation for the inconsistency in previous research findings is that the cancer risk elevation in people with affective disorders may be site-specific. In other words, we have to look into the mechanism for the development of a specific cancer rather than assuming a generalised elevation of cancer risk. For example,

*These authors contributed equally to the work. one Danish study ${ }^{8}$ found increased risk for tobacco-related cancers (such as lungs, larynx, buccal cavity, oesophagus, pancreas, kidney and bladder cancers) in patients with depression and no risk elevation for non-tobacco-related cancers such as colon and breast cancers. From a public health perspective, research tapping into modifiable lifestyle factors and subsequent cancer risks in specific sites would have a greater impact than research into an overall cancer-risk elevation. This way, the scale of the problems in relation to lifestyle-associated cancers can be estimated, and public health measures can be enacted to change or to encourage healthier lifestyle behaviours. This means we would need a large cohort to investigate mechanism- and site-specific cancer incidence. Several previous studies were limited by their short time frames and small sample sizes for incident site-specific cancer events, ${ }^{7,16}$ and thus, the risks for site-specific cancers were not able to be estimated with sufficient statistical power. We found very few studies exploring lifestyle factors in relation to cancer risk in patients with affective disorders; even so, it is well-recognised that patients with affective disorders tend to live more unhealthy lifestyles (for example smoking and drinking alcohol) ${ }^{4}$ than the general population does. In Taiwan, $29.0 \%$ of patients with bipolar disorder have been shown to have a smoking habit ${ }^{17}$ and $8.6 \%$ to be alcoholics. ${ }^{18}$ The prevalence of substance use disorder has been found to be higher among patients with a diagnosis of major depressive disorder than among bipolar patients. ${ }^{19}$ The aim of this study was to estimate the risk of alcohol- and tobacco-related cancers in a large Taiwanese cohort with affective disorders.

\section{Method}

\section{Data sources}

Details of the data source have been described previously. ${ }^{20}$ Briefly, Taiwan introduced a single-payer National Health Insurance 
Program on 1 March 1995 and $98 \%$ of the 23 million Taiwanese population were enrolled. In 1996, the National Health Research Institute in Taiwan established the National Health Insurance Research Database. The database includes medical claim files representative of the entire population in Taiwan. All investigators signed an agreement guaranteeing patient confidentiality before using the database.

We used the Psychiatric Inpatient Medical Claims database, a subset of the National Health Insurance Research Database, comprising a cohort of people admitted to hospital for any psychiatric disorder between 1996 and $2008(n=187117)$. The database included patients with at least one psychiatric in-patient record and one discharge diagnosis of mental illness coded according to the ICD-9 as codes $290-319 .^{21}$ The database includes patient demographic characteristics, diagnoses, medical expenditure and prescription claims data. Information that could be used to identify beneficiaries and medical care providers was scrambled by the National Health Research Institute.

\section{Identification of the study cohort}

We conducted a prospective cohort study to examine cancer incidence in people with affective disorder. The data on individuals analysed in this study were identified through the Psychiatric Inpatient Medical Claims database (ICD-9-CM code: 296). Individuals who had a psychiatric admission to hospital between 1 January 1997 and 31 December 2002 with the diagnosis of affective disorders (ICD-9-CM code: 296) were included. Those who had been admitted during 1996 were excluded: we used a 1 -year exclusion period to capture 'first admitted' patients. The earliest admission date for each cohort member was defined as the index date. Individuals were excluded if they: (a) did not have information on gender or age; (b) received a diagnosis of cancer before the index date; or (c) received any diagnosis of schizophrenia (ICD-9 CM code: 295) during 1996-2010.

Affective disorders were further categorised as bipolar disorder (ICD-9-CM codes: 296.0, 296.1 and 296.4-296.9, excluding 296.82) $(n=10207)$ or major depressive disorder (ICD-9-CM codes: 296.2, 296.3 and 296.82) $(n=9826)$. The detailed patient identification algorithm is shown in Fig. 1. All patients $(n=20033)$ had been monitored from the index date to cancer diagnosis, death or until the end of 2010, whichever came first.

\section{Identification of cancer events}

In the database, cancer was defined as a 'catastrophic illness', and thus, the status of 'catastrophic illness' enabled the co-payment for the treatment of the disease to be waived. Cytological and/or other pathological reports or evidence supportive of malignancy were required to apply for a catastrophic illness certificate for cancer. Cancer events in the present study were identified by the link of the study cohort with the catastrophic illness database (ICD-9-CM codes: 140-208). We further identified cancers sites that were tobacco- or alcohol-related. Although we did not have tobacco or alcohol use in the data-set, we identified alcohol- and/or tobacco-related cancers based on the most updated categorisation defined by the World Health Organization (WHO) International Agency for Research on Cancer Monographs. ${ }^{22-24}$ Accordingly, tobacco-related cancer sites (ICD-9-CM codes) included the lung (162), oral cavity and pharynx (140-149), nasal cavity and paranasal sinuses (160), larynx (161), oesophagus (150), stomach (151), pancreas (157), liver (155), kidney (189), urinary bladder (188), uterine cervix (180), as well as myeloid leukaemia $(205) .^{22}$ Alcohol-related cancer sites included the oral cavity, pharynx, larynx, oesophagus, liver, colorectum (153-154) and

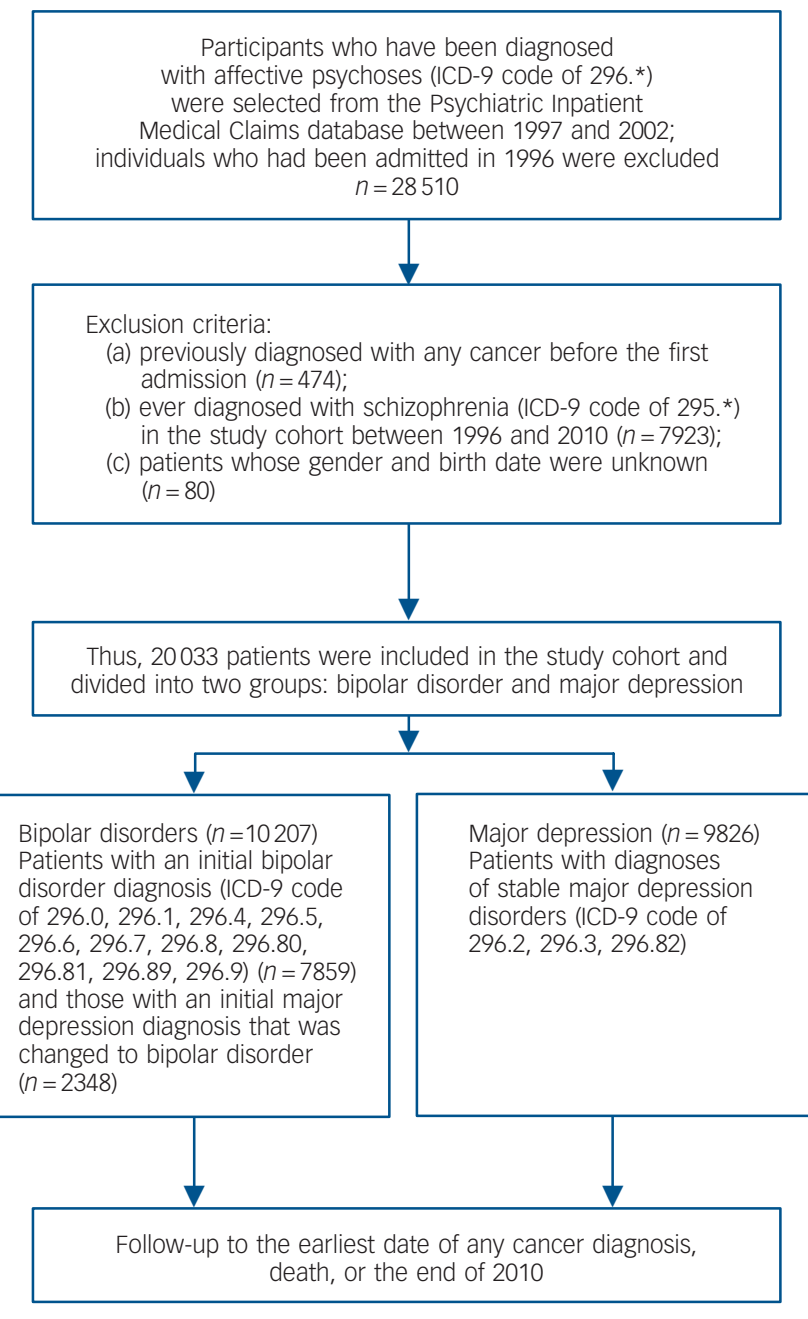

Fig. 1 Flow chart of patient selection.

female breast (174). ${ }^{23,24}$ This categorisation has been utilised by previous studies to examine alcohol- and tobacco-related cancer risk. ${ }^{7,25}$ We also identified gender-specific cancers (ICD-9-CM codes): for females, breast (174), cervix (180) and body of the uterus (182) and for males, prostate cancer (185).

\section{Estimation of standardised incidence ratios}

The SAS System for Windows (Version 9.2) was used to manage and analyse the data. We used standardised incidence ratios (SIRs) to determine whether the occurrence of cancer in people with affective disorders was higher or lower than in the general population. Standardised incidence ratios were computed as the ratio of the observed number to the expected number of cancer events in the study cohort. The expected number of cancer events was calculated by multiplying the number of individuals at risk by the national cancer incidence rate and the length of follow-up time (years). The national incidence rates of all, and site-specific, cancers were retrieved from the cancer statistics website of the Health Promotion Administration, Ministry of Health and Welfare (https://cris.hpa.gov.tw/). The data has been recorded and updated annually (https://tcr.cph.ntu.edu.tw/main.php?Page=N1) with the most recent data available at the time of this study having been for the year 2008. We hence took the average incident rates of 1999-2008 to estimate the expected number of cancer events (i.e. the first 2 years and the last 2 years of the follow-up period were 
excluded for the calculation of average cancer incident rates). Confidence intervals of SIRs were estimated using the BoiceMonson method. ${ }^{26}$

\section{Sensitivity analysis}

To assess the robustness of our study results, we conducted numerous sensitivity analyses. First, the ICD-9 code 296.9 (affective disorder, unspecified) could contain bipolar, unipolar disorder or other atypical/unspecified conditions related to affective disorders. Therefore, we excluded people in this residual category (affective disorder, unspecified (296.9)) to assess if the results remained the same. Second, we excluded individuals with an alcohol-related diagnosis (ICD-9-CM codes: 291, 303, 305.0, 980.0) $(n=1514,7.56 \%)$. As alcoholism is a risk factor for cancer, ${ }^{23}$ including people with alcohol-related diagnoses may inflate the cancer risk estimates for affective disorders. Third, we excluded people who were admitted because of affective disorder during 1996-1998, and restricted our analyses to those who received in-patient treatment during 1999-2002 alone. It is very likely that those who were admitted for the treatment of affective disorders during 1996-1998 could have had their first admission before the inception year (1996) of the National Health Insurance Program. A recent study ${ }^{27}$ reported that in Taiwan, the 1-year relapse rate for individuals with bipolar disorder who had a history of in-patient treatment was $55 \%$. In an 18 -month follow-up study conducted on patients with major depression, only about a quarter were never readmitted, and $49 \%$ experienced residual symptoms or recurrences. ${ }^{28}$ Therefore, by utilising a 3 -year exclusion period, we were better able to capture the 'true first admitted' patients with affective disorder than when using the whole cohort collected during the period between 1997 and 2002.

\section{Results}

\section{Incidence of cancer in patients with bipolar disorder and major depression}

The incidences of cancer in the affective disorder cohorts were 4.22 and 6.27 per 1000 person-years for people with bipolar disorder and major depressive disorders, respectively; the corresponding figure in the general population of Taiwan was 3.03 per 1000 person-years.

Elevated cancer risks were found in people with bipolar disorder and major depression and in both genders; the overall risk elevation, however, was higher in people with major depressive disorders (SIR 2.01, 95\% CI 1.85-2.19) than in those with bipolar disorder (SIR 1.39, 95\% CI 1.26-1.53) (Table 1). In-patient care for affective disorders at a young age was associated with a higher risk of subsequent cancer than at an older age; for those who received in-patient care for affective disorder at 70 years of age or older, the risk of cancer was lower than in the general population (Table 1).

\section{Site-specific cancers in people with bipolar disorder and depression}

Table 2 presents site-specific cancers stratified by lifestyle behaviours for affective disorders. For bipolar and major depressive disorders, cancer sites that were related to both tobacco and alcohol use had the highest incidence rates when compared with cancer sites that were related to either tobacco or alcohol use or cancer sites that were not alcohol- or tobacco-related.

For bipolar disorder, the elevated cancer risk was significant, yet smaller, for cancer sites that were associated with single substance use (either tobacco or alcohol). It is remarkable that, for women with bipolar disorder, prominent elevated risk of breast

\begin{tabular}{|c|c|c|c|c|c|c|c|}
\hline & $n$ & $\begin{array}{l}\text { Follow-up duration, } \\
\text { years: mean (s.d.) }\end{array}$ & $\begin{array}{l}\text { Cancer cases } \\
\text { observed, } n\end{array}$ & $\begin{array}{c}\text { Crude } \\
\text { incidence }^{a}\end{array}$ & $\begin{array}{c}\text { Expected } \\
\text { incidence, } n\end{array}$ & $\operatorname{SIR}^{\mathrm{b}}(95 \% \mathrm{CI})$ & $P$ \\
\hline $\begin{array}{l}\text { Bipolar disorder } \\
\text { Gender }\end{array}$ & 10207 & 9.49 (3.12) & 409 & 422.24 & 294.24 & 1.39 (1.26-1.53) & $<0.001$ \\
\hline Female & 5184 & $9.86(2.84)$ & 181 & 354.11 & 135.07 & $1.34(1.16-1.55)$ & $<0.001$ \\
\hline Male & 5023 & 9.11 (3.33) & 228 & 498.26 & 159.44 & $1.43(1.26-1.63)$ & $<0.001$ \\
\hline \multicolumn{8}{|l|}{ Age, years ${ }^{c}$} \\
\hline$<20$ & 810 & $10.32(2.25)$ & 6 & 71.78 & 1.14 & $5.25(2.36-11.69)$ & $<0.001$ \\
\hline $20-29$ & 2459 & $10.03(3.12)$ & 28 & 113.53 & 7.82 & $3.58(2.47-5.18)$ & $<0.001$ \\
\hline $30-39$ & 2470 & $9.86(2.90)$ & 71 & 291.53 & 26.79 & $2.65(2.10-3.35)$ & $<0.001$ \\
\hline $40-49$ & 1961 & $9.48(3.10)$ & 89 & 478.74 & 52.66 & $1.69(1.37-2.08)$ & $<0.001$ \\
\hline $50-59$ & 1163 & 9.18 (3.33) & 81 & 758.69 & 56.25 & $1.44(1.16-1.79)$ & $<0.001$ \\
\hline $60-69$ & 775 & $8.29(3.64)$ & 93 & 1447.53 & 62.84 & $1.48(1.21-1.81)$ & $<0.001$ \\
\hline 70-79 & 470 & $7.17(3.96)$ & 38 & 1127.63 & 52.78 & $0.72(0.52-0.99)$ & 0.0428 \\
\hline$\geqslant 80$ & 99 & $4.44(3.71)$ & 3 & 682.50 & 8.11 & $0.37(0.12-1.14)$ & 0.0709 \\
\hline $\begin{array}{l}\text { Major depression } \\
\text { Gender }\end{array}$ & 9826 & $8.56(3.42)$ & 527 & 626.56 & 262.19 & $2.01(1.85-2.19)$ & $<0.001$ \\
\hline Female & 5147 & $9.21(3.13)$ & 280 & 590.67 & 126.13 & $2.22(1.97-2.50)$ & $<0.001$ \\
\hline Male & 4679 & $7.84(3.58)$ & 247 & 673.33 & 130.69 & $1.89(1.67-2.14)$ & $<0.001$ \\
\hline \multicolumn{8}{|l|}{ Age, years ${ }^{c}$} \\
\hline$<20$ & 468 & $9.41(2.26)$ & 1 & 22.71 & 0.60 & $1.66(0.23-11.81)$ & 0.6069 \\
\hline $20-29$ & 2102 & $9.14(2.28)$ & 15 & 78.08 & 6.10 & $2.46(1.48-4.08)$ & $<0.001$ \\
\hline 30-39 & 1787 & $9.25(2.28)$ & 53 & 320.63 & 18.15 & $2.92(2.23-3.82)$ & $<0.001$ \\
\hline $40-49$ & 1796 & $9.16(3.22)$ & 118 & 717.27 & 46.64 & $2.53(2.11-3.03)$ & $<0.001$ \\
\hline $50-59$ & 1189 & 8.99 (3.30) & 102 & 954.24 & 56.04 & $1.82(1.50-2.20)$ & $<0.001$ \\
\hline $60-69$ & 1060 & $8.15(3.82)$ & 123 & 1423.78 & 84.25 & $1.46(1.22-1.74)$ & $<0.001$ \\
\hline 70-79 & 1121 & $6.20(3.90)$ & 96 & 1381.26 & 109.09 & $0.88(0.72-1.08)$ & 0.2261 \\
\hline$\geqslant 80$ & 303 & 4.09 (3.39) & 19 & 1533.16 & 22.89 & $0.83(0.53-1.30)$ & 0.4047 \\
\hline
\end{tabular}




\begin{tabular}{|c|c|c|c|c|c|c|}
\hline \multirow{2}{*}{$\begin{array}{l}\text { Site of cancer } \\
\text { (modified ICD-9-CM) }\end{array}$} & \multicolumn{3}{|c|}{ Bipolar disorder } & \multicolumn{3}{|c|}{ Major depression } \\
\hline & Observed, $n$ & $\mathrm{SIR}^{\mathrm{a}}(95 \% \mathrm{Cl})$ & $P$ & Observed, $n$ & $\mathrm{SIR}^{\mathrm{a}}(95 \% \mathrm{Cl})$ & $P$ \\
\hline Total (140-208) & 409 & $1.39(1.26-1.53)$ & $<0.001$ & 527 & $2.01(1.85-2.19)$ & $<0.001$ \\
\hline Tobacco-, alcohol-related & 124 & $1.75(2.21-3.44)$ & $<0.001$ & 145 & $2.32(1.97-2.73)$ & $<0.001$ \\
\hline Buccal cavity (140-149) & 58 & $2.97(2.29-3.84)$ & $<0.001$ & 43 & $2.50(1.85-3.37)$ & $<0.001$ \\
\hline Oesophagus (150) & 17 & $2.65(1.65-4.26)$ & $<0.001$ & 21 & $3.72(2.42-5.69)$ & $<0.001$ \\
\hline Larynx (161) & 3 & $1.28(0.41-3.98)$ & 0.6640 & 9 & $4.37(2.28-8.05)$ & $<0.001$ \\
\hline Liver (155) & 46 & $1.08(0.81-1.44)$ & 0.6179 & 72 & $1.91(1.52-2.41)$ & $<0.001$ \\
\hline Tobacco-related only & 107 & $1.29(1.07-1.56)$ & 0.0083 & 143 & $1.94(1.65-2.29)$ & $<0.001$ \\
\hline Lung (162) & 40 & $1.14(0.83-1.55)$ & 0.4147 & 51 & $1.65(1.25-2.17)$ & $<0.001$ \\
\hline Nasal cavity and paranasal sinuses (160) & 2 & $2.80(0.70-11.20)$ & 0.1279 & 0 & - & - \\
\hline Pancreas (157) & 4 & $0.77(0.29-2.04)$ & 0.5927 & 15 & $3.26(1.97-5.41)$ & $<0.001$ \\
\hline Stomach (151) & 22 & $1.42(0.94-2.16)$ & 0.0953 & 27 & $1.99(1.36-2.89)$ & $<0.001$ \\
\hline Kidney (189) & 7 & $0.91(0.44-1.92)$ & 0.8122 & 17 & $2.52(1.57-4.05)$ & $<0.001$ \\
\hline Bladder (188) & 9 & $1.08(0.56-2.08)$ & 0.8177 & 11 & $1.50(0.83-2.71)$ & 0.1766 \\
\hline Uterine cervix (180) & 19 & $1.82(1.16-2.85)$ & 0.0081 & 10 & $1.02(0.55-1.90)$ & 0.9405 \\
\hline Myeloid leukemia $(205)^{b}$ & 4 & - & - & 12 & - & - \\
\hline Alcohol-related only & 85 & $1.25(1.01-1.55)$ & 0.0366 & 129 & $2.11(1.77-2.50)$ & $<0.001$ \\
\hline Colorectal cancer (153-154) & 39 & $0.98(0.72-1.35)$ & 0.9139 & 62 & $1.77(1.38-2.28)$ & $<0.001$ \\
\hline Breast (174), female only & 46 & $1.64(1.23-2.18)$ & $<0.001$ & 67 & $2.55(2.01-3.24)$ & $<0.001$ \\
\hline Non-tobacco, non-alcohol-related & 93 & $1.11(0.91-1.37)$ & 0.2967 & 110 & $1.51(1.68-2.12)$ & $<0.001$ \\
\hline Skin cancer (172-173) & 6 & $0.66(0.30-1.47)$ & 0.3033 & 7 & $0.87(0.42-1.83)$ & 0.7188 \\
\hline Brain tumour (191) & 4 & $1.53(0.57-4.08)$ & 0.3919 & 9 & $3.91(2.03-7.51)$ & $<0.001$ \\
\hline Thyroid cancer (193) & 11 & $1.65(0.92-2.99)$ & 0.0920 & 12 & $2.05(1.16-3.61)$ & 0.0112 \\
\hline Other and unspecified sites & 51 & $1.02(0.78-1.35)$ & 0.8661 & 53 & $1.21(0.92-1.58)$ & 0.1678 \\
\hline Gender-specific & 86 & $1.6(1.29-1.97)$ & $<0.001$ & 106 & $2.16(1.79-2.61)$ & $<0.001$ \\
\hline Breast (174), female only & 46 & $1.64(1.23-2.18)$ & $<0.001$ & 67 & $2.55(2.01-3.24)$ & $<0.001$ \\
\hline Cervical cancer (180), female only & 19 & $1.82(1.16-2.85)$ & 0.0081 & 10 & $1.02(0.55-1.90)$ & 0.9405 \\
\hline Body of uterus (182), female only & 9 & $2.10(1.09-4.03)$ & 0.0231 & 3 & $0.75(0.24-2.32)$ & 0.6127 \\
\hline Prostate (185), male only & 12 & $1.09(0.62-1.92)$ & 0.7598 & 26 & $2.88(1.96-4.23)$ & $<0.001$ \\
\hline
\end{tabular}

and gynaecological cancers was found. Elevated cancer risks were particularly high for the following specific cancer sites (SIR $\geqslant 2.5$ ): buccal cavity, oesophagus and nasal cavity/paranasal sinuses.

For people with major depressive disorder, a more generalised increase in cancer risk was observed across all cancer sites; except for skin, bladder and gynaecological cancers (i.e. cervical and uterine cancers). Elevated cancer risks were particularly high for specific cancer sites ( $\mathrm{SIR} \geqslant 2.5$ ); these were the buccal cavity, oesophagus, larynx, pancreas, kidney, brain, breast and prostate.

\section{Sensitivity analysis}

All three sensitivity analyses did not differ significantly from the results using the full sample. When patients coded under ICD-9 296.9 (affective disorder, unspecified) were excluded, the SIRs for all other patients, those with bipolar disorder and those with major depression were $1.65(P<0.001), 1.35(P<0.001)$ and $1.96(P<0.001)$, respectively. In the analysis excluding individuals with alcohol-related disorders, the corresponding SIRs were 1.50 $(P<0.001), 1.24(P<0.001)$ and $1.80(P<0.001)$, respectively. The corresponding figures for the results using the 1999-2002 subset sample (i.e. 3-year exclusion period) were 1.72 $(P<0.001), 1.38(P<0.001)$, and $2.05(P<0.001)$

\section{Discussion}

\section{Main findings}

We found significantly increased cancer risk in a large cohort of people who had been admitted to hospital for affective disorders in Taiwan. However, the increase in cancer risk was not found among individuals whose first psychiatric admission to hospital was at 70 years of age or older. The extent of risk elevation was more pronounced among people with major depressive disorders than among those with bipolar disorders. The risk of site-specific cancers suggests that, for people with bipolar disorder, the elevated risks were generally limited to female-hormone-regulated cancers such as breast, uterine and cervical cancers and cancers associated with tobacco and alcohol use; whereas for individuals with major depression, a more generalised increase in cancer risk was found.

The elevated cancer risk among patients ever admitted to hospital for affective disorders was more pronounced in tobaccoand/or alcohol-related cancers and in those who received in-patient care for affective disorders at a young age. Because the prevalence of smoking and alcohol use is much higher in people with affective disorders than among the general population, ${ }^{29-31}$ lifestyle behaviours are likely to represent an important factor in the development of cancer in people with affective disorders.

\section{Findings from other studies}

One Danish study ${ }^{8}$ showed that the increased cancer risk in people with affective disorders was only found in cancers associated with tobacco use; no elevation was noted in non-tobacco-related cancer sites, unlike the findings of the present study. Therefore, the underlying mechanisms of the relationship between affective disorders and cancer risk may vary between different populations.

\section{Age-related differences in incidence}

Our results showed that cancer incidence in affective disorders was age-related. The younger a patient was when receiving their first 
in-patient treatment for affective disorders, the higher their subsequent risk of cancer. However, the risk of cancer for individuals whose first admission for affective disorder occurred when they were 70 years of age or older was paradoxically lower than that for the general population. To the best of our knowledge, no study to date has reported age-stratified cancer incidences among individuals with affective disorder. This is probably because very few cohorts had adequate sample sizes to conduct age-stratified analyses. Our findings suggest a common genetic liability for cancer and affective disorders as early-onset diseases tend to have a genetic origin. This hypothesis, however, has not been formally validated. One other explanation is the high prevalence of harmful health behaviours, such as cigarette smoking and alcohol use, in young people with affective disorders in Taiwan. ${ }^{32}$

\section{Findings relating to major depression}

The subsequent cancer risks in people with affective disorders were more pronounced in those with major depression than in those with bipolar disorder. In individuals with major depression, the average risk of cancers related to both alcohol and tobacco use (i.e. oral cavity and oesophageal cancers) reached 2.32, strongly indicating the role of health behaviours in exacerbating cancer risk. As Taiwanese people with major depression have been reported to have a higher prevalence of substance use disorder than those with bipolar disorder, ${ }^{19}$ it is likely that the increased cancer risk in those with depression is related to the higher prevalence of unhealthy lifestyle behaviours among this population. However, the elevated risk was not limited to tobaccoand alcohol-related cancers; for cancers that were not related to tobacco or alcohol use, a $51 \%$ excess risk was found in those with major depressive disorders compared with the general population. This figure is much higher than findings from Western countries. A meta-analysis pooling studies from 1990 to 2005, all conducted in the West, yielded a slightly and marginally increased cancer risk in patients with depressive disorders (adjusted odds ratio 1.12, 95\% CI 0.99-1.26). ${ }^{33}$ The high incidence of cancer in people with major depression adds to the overall high risk status of these individuals in Taiwan. The prevalence of depression in Taiwan and other Asian countries is much lower than that reported in Western countries. ${ }^{34}$ Thus, our study results highlight the need to pay particular attention to the physical health of people with depressive disorders in Taiwan.

The increased risk of brain cancer in people with major depression was extremely high. Because depression can be an early manifestation of brain cancer, this finding highlights the importance of considering physical disease as a possible differential diagnosis for individuals with depressive symptoms.

\section{Findings relating to bipolar disorder}

Although the extent of cancer risk elevation was not as prominent in people with bipolar disorder compared with those with depressive disorders, we observed a significant increase in female-hormone-regulated cancers such as breast, cervical and uterine cancers, in addition to tobacco- and alcohol-related cancers, among people with bipolar disorder. One possible mechanism for this increase is the elevated prolactin levels subsequent to the use of psychotropic medications in patients with bipolar disorder. ${ }^{12}$ In fact, increased risk of cancer influenced by female hormones, such as breast cancer, was also found in people with schizophrenia ${ }^{12,35}$ even though the overall cancer risk is widely believed to be lower in individuals with schizophrenia compared with the general population. ${ }^{36-38}$ Active screening for female-hormone-regulated cancers in people with bipolar disorder should be integrated into clinical practice.

\section{Strengths and limitations}

One key strength of the present study is its large sample size. The study included more than 20000 people with mood disorders and we assessed site-specific cancers to ascertain whether the risk elevation was associated with tobacco- or alcohol-related cancer. Nonetheless, several limitations are associated with the study. First, because we used the claims database, no information on smoking and drinking behaviours were included; thus, we could only identify cancer sites that were associated with tobacco and alcohol use. These are proxy measures rather than true assessments of unhealthy lifestyle behaviours.

Second, we only included people with mood disorders who were ever admitted to hospital and this population might not be representative of individuals with mood disorders who have not received in-patient treatment.

Third, cancer events in patients with affective disorders were measured based on the catastrophic illness database. This database is linked to the National Health Insurance Data. The process of applying for catastrophic disease registration is quite rigorous and the validity of case identification is very high. ${ }^{39,40}$ Hence, the catastrophic illness database has been widely used in previous studies to ascertain cancer events. ${ }^{39,40}$ The National Cancer Registry database is derived from a population-based cancer registry with the goal of monitoring the incidence of cancer among the general population of Taiwan. This Registry is not linked to the National Health Insurance Research Database, but can be used to calculate population incidence rates. The number of cancer events identified by the Catastrophic Illness database is likely to be lower than the number identified in the National Cancer Registry database. Thus, the SIRs were likely to be underestimated in this study. In other words, we provided a conservative estimation of SIRs in our sample; the true figures of cancer incidence in people with affective disorders could be even higher.

Fourth, this study excluded individuals with any diagnosis of cancer before the index date $(n=474)$. However, some of these excluded people may have experienced their onset of affective disorder prior to the inception year of the National Health Insurance Program. Therefore, it is likely that this contributed to a further underestimation of the effect size reported by this study.

Finally, individual variations in healthcare utilisation could have influenced the estimated association between affective disorder and subsequent cancer incidence. However, because Taiwan's National Health Insurance covers $99 \%$ of the population for both psychiatric and medical in-patient and out-patient services, it is not likely that patterns of healthcare utilisation would affect our results significantly.

\section{Implications}

Given our findings, we recommend particular attention is focused on lifestyle behaviours and the associated cancer risks in people with affective disorders, especially those who have ever been admitted to hospital for affective disorders at a young age. To achieve better healthcare quality, caring for people with affective disorders should be holistic. Namely, in addition to patient mental health, physical conditions should be monitored proactively. 
Yen-Ni Hung, PhD, School of Gerontology Health Management and Master Program in Long-Term Care, College of Nursing, Taipei Medical University, Department of Nursing, School of Nursing, National Yang-Ming University and Department of Education and Research, Taipei City Hospital, Taipei; Shu-Yu Yang, PhD, Taipei City Psychiatric Center, Taipei City Hospital, Taipei and Graduate Institute of Clinical Pharmacy, College of Pharmacy, Kaohsiung Medical University, Kaohsiung, Taiwan; Ming-Chyi Huang, MD, PhD, Taipei City Psychiatric Center, Taipei City Hospital and Department of Psychiatry, School of Medicine, Taipei Medical University, Taipei; For-Wey Lung, MD, PhD, Taipei City Psychiatric Center, Taipei City Hospital, Taipel; Shih-Ku Lin, MD,Taipei City Psychiatric Center, Taipei City Hospital and Department of Psychiatry, School of Medicine, Taipei Medical University, Taipei; Kuan-Yu Chen MD, PhD, Taipei City Psychiatric Center, Taipei City Hospital, Taipei; Chian-Jue Kuo, MD, PhD, Taipei City Psychiatric Center, Taipei City Hospital and Department of MD, PhD, Taipei City Psychiatric Center, Taipei City Hospital and Department of
Psychiatry, School of Medicine, Taipei Medical University and Psychiatric Research Center, Taipei Medical University Hospital, Taipei, Taiwan; Ying-Yeh Chen, MD, SCD, Taipei City Psychiatric Center, Taipei City Hospital and Institute of Public Health and Department of Public Health, National Yang-Ming University, Taipei, Taiwan.

Correspondence: Ying-Yeh Chen, MD, SCD, Department of General Psychiatry, Taipei City Psychiatric Center, 309 Sung-Te Road, Taipei, 110, Taiwan. Email:ychen@tpech.gov.tw

First received 6 Sep 2013, final revision 13 Jan 2014, accepted 28 Mar 2014

\section{Funding}

This research was supported by grants from the National Science Council (NSC102-2628-B532-001-MY3) of Taiwan and Taipei City Hospital (10101-62-008, 10101-62-055), Taipei, Taiwan.

\section{References}

1 Muller-Oerlinghausen B, Berghofer A, Bauer M. Bipolar disorder. Lancet 2002 359: 241-7.

2 Bai YM, Su TP, Chen MH, Chen TJ, Chang WH. Risk of developing diabetes mellitus and hyperlipidemia among patients with bipolar disorder, major depressive disorder, and schizophrenia: a 10-year nationwide populationbased prospective cohort study. J Affect Disord 2013; 150: 57-62.

3 Lozano R, Naghavi M, Foreman K, Lim S, Shibuya K, Aboyans V, et al. Global and regional mortality from 235 causes of death for 20 age groups in 1990 and 2010: a systematic analysis for the Global Burden of Disease Study 2010 Lancet 2012; 380: 2095-128.

4 Jane-Llopis E, Matytsina I. Mental health and alcohol, drugs and tobacco: a review of the comorbidity between mental disorders and the use of alcohol tobacco and illicit drugs. Drug Alcohol Rev 2006; 25: 515-36.

5 Dreyer L, Winther JF, Andersen A, Pukkala E. Avoidable cancers in the Nordic countries. Alcohol consumption. APMIS Suppl 1997; 76: 48-67.

6 Dreyer L, Winther JF, Pukkala E, Andersen A. Avoidable cancers in the Nordic countries. Tobacco smoking. APMIS Suppl 1997; 76: 9-47.

7 Whitley E, Batty GD, Mulheran PA, Gale CR, Osborn DP, Tynelius P, et al. Psychiatric disorder as a risk factor for cancer: different analytic strategies produce different findings. Epidemiology 2012; 23: 543-50.

8 Dalton SO, Mellemkjaer L, Olsen JH, Mortensen PB, Johansen C. Depression and cancer risk: a register-based study of patients hospitalized with affective disorders, Denmark, 1969-1993. Am J Epidemiol 2002; 155: 1088-95.

9 Beumer W, Gibney SM, Drexhage RC, Pont-Lezica L, Doorduin J, Klein HC, et al. The immune theory of psychiatric diseases: a key role for activated microglia and circulating monocytes. J Leukoc Biol 2012; 92: 959-75.

10 Tsai SY, Chung KH, Wu JY, Kuo CJ, Lee HC, Huang SH. Inflammatory markers and their relationships with leptin and insulin from acute mania to full remission in bipolar disorder. J Affect Disord 2012; 136: 110-6.

11 BarChana M, Levav I, Lipshitz I, Pugachova I, Kohn R, Weizman A, et al. Enhanced cancer risk among patients with bipolar disorder. J Affect Disord 2008; 108: 43-8.

12 McGinty EE, Zhang $Y$, Guallar E, Ford DE, Steinwachs D, Dixon LB, et al. Cancer incidence in a sample of Maryland residents with serious mental illness. Psychiatr Serv 2012; 63: 714-7.

13 Chen $\mathrm{YH}$, Lin HC. Increased risk of cancer subsequent to severe depressiona nationwide population-based study. J Affect Disord 2011; 131: 200-6.

14 Rosenshfir E, Shemesh P, Aizenberg D, Barak Y. Reduced risk of cancer in patients with bipolar affective disorder. Neurosci \& Med 2011; 2: 57-60.

15 Liang JA, Sun LM, Muo CH, Sung FC, Chang SN, Kao CH. The analysis of depression and subsequent cancer risk in Taiwan. Cancer Epidemiol Biomarkers Prev 2011; 20: 473-5.
16 Possel P, Adams E, Valentine JC. Depression as a risk factor for breast cancer: investigating methodological limitations in the literature. Cancer Causes Control 2012; 23: 1223-9.

17 Tsai SY, Yang YY, Kuo CJ, Chen CC, Leu SJ. Effects of symptomatic severity on elevation of plasma soluble interleukin-2 receptor in bipolar mania. J Affect Disord 2001; 64: 185-93.

18 Hsieh $\mathrm{MH}$, Tang $\mathrm{CH}$, Hung ST, Lee IH, Lin YJ, Yang YK. Comorbid prevalence of alcohol dependence, substance abuse, and external cause of injury in patients with bipolar disorder in a nationwide representative sample in Taiwan. Bipolar Disord 2012; 14: 677-9.

19 Bai YM, Lin CC, Hu PG, Yeh HS. Risk factors for substance use disorders among inpatients with major affective disorders in Taiwan Chinese. Gen Hosp Psychiatry 1998; 20: 377-80

20 Gau SS, Chao PF, Lin YJ, Chang CJ, Gau CS. The association between carbamazepine and valproate and adverse cutaneous drug reactions in patients with bipolar disorder: a nested matched case-control study. J Clin Psychopharmacol 2008; 28: 509-17.

21 World Health Organization. International Statistical Classification of Diseases and Related Health Problems (ICD-9). WHO, 1978.

22 IARC Working Group on the Evaluation of Carcinogenic Risks to Humans Tobacco smoke and involuntary smoking. IARC Monogr Eval Carcinog Risks Hum 2004; 83: 1-1438.

23 Baan R, Straif K, Grosse Y, Secretan B, El Ghissassi F, Bouvard V, et al. Carcinogenicity of alcoholic beverages. Lancet Oncol 2007; 8: 292-3.

24 IARC Working Group on the Evaluation of Carcinogenic Risks to Humans. Alcohol consumption and ethyl carbamate. IARC Monogr Eval Carcinog Risks Hum 2010; 96: 3-1383.

25 Woo HD, Kim J. Dietary flavonoid intake and smoking-related cancer risk: a meta-analysis. PLOS One 2013; 8: e75604.

26 Rothman KJ, Greenland S. Modern Epidemiology (2nd edn). Lippincott Williams \& Wilkins, 1998

27 Tang $\mathrm{CH}$, Hsieh MH, Hung ST, Lee IH, Lin YJ, Yang YK. One-year post-hospital medical costs and relapse rates of bipolar disorder patients in Taiwan: a population-based study. Bipolar Disord 2010; 12: 859-65.

28 Vuorilehto MS, Melartin TK, Isometsa ET. Course and outcome of depressive disorders in primary care: a prospective 18-month study. Psychol Med 2009; 39: 1697-707.

29 Heffner JL, Strawn JR, DelBello MP, Strakowski SM, Anthenelli RM. The co?occurrence of cigarette smoking and bipolar disorder: phenomenology and treatment considerations. Bipolar Disord 2011; 13: 439-53.

30 Lasser K, Boyd JW, Woolhandler S, Himmelstein DU, McCormick D, Bor DH. Smoking and mental illness. JAMA 2000; 284: 2606-10.

31 Conway KP, Compton W, Stinson FS, Grant BF. Lifetime comorbidity of DSM-IV mood and anxiety disorders and specific drug use disorders: results from the National Epidemiologic Survey on Alcohol and Related Conditions. J Clin Psychiatry 2006; 67: 247-57.

32 Wang PW, Lin HC, Yeh YC, Liu TL, Yen CF. The relation of substance use with different levels of depressive symptoms and the moderating effect of sex and age in Taiwanese adolescents. Compr Psychiatry 2012; 53: 1013-20.

33 Oerlemans ME, van den Akker M, Schuurman AG, Kellen E, Buntinx F. A meta-analysis on depression and subsequent cancer risk. Clin Pract Epidemiol Ment Health 2007; 3: 29.

34 Horwath E, Weissman MM. Epidemiology of depression and anxiety disorders. In Textbook in Psychiatric Epidemiology (eds MT Tsuang, M Tohen and GEP Zahner): 317-44. Wiley-Liss, 1995.

35 Catts V, Catts S, O'toole B, Frost A. Cancer incidence in patients with schizophrenia and their first?degree relatives-a meta?analysis. Acta Psychiatr Scand 2008; 117: 323-36.

36 Lichtermann D, Ekelund J, Pukkala E, Tanskanen A, Lonnqvist J. Incidence of cancer among persons with schizophrenia and their relatives. Arch Gen Psychiatry 2001; 58: 573

37 Barak Y, Achiron A, Mandel M, Mirecki I, Aizenberg D. Reduced cancer incidence among patients with schizophrenia. Cancer 2005; 104: 2817-21.

38 Hippisley-Cox J, Vinogradova $Y$, Coupland C, Parker C. Risk of malignancy in patients with schizophrenia or bipolar disorder: nested case-control study. Arch Gen Psychiatry 2007; 64: 1368-76.

39 Lin GM, Chen YJ, Kuo DJ, Jaiteh LE, WU YC, Lo TS, et al. Cancer incidence in patients with schizophrenia or bipolar disorder: a nationwide populationbased study in Taiwan, 1997-2009. Schizophr Bull 2013; 39: 407-16.

40 Chen YJ, Wu CY, Chen TJ, Shen JL, Chu SY, Wang CB, et al. The risk of cancer in patients with psoriasis: a population-based cohort study in Taiwan. J Am Acad Dermatol 2011; 65: 84-91. 